\title{
Increased activation product of complement 4 protein in plasma of individuals with schizophrenia
}

\author{
Agnieszka Kalinowski (iD ${ }^{1,2}$, Joanna Liliental ${ }^{3,4,5}$, Lauren A. Anker (iD ${ }^{1,2}$, Omer Linkovski (iD ${ }^{1,6}$, Collin Culbertson ${ }^{7}$, Jacob N. Hall $\mathbb{D}^{7,8}$, \\ Reenal Pattni ${ }^{1,9}$, Chiara Sabatti ${ }^{10}$, Douglas Noordsy ${ }^{1}$, Joachim F. Hallmayer ${ }^{1,2}$, Elizabeth D. Mellins (D) ${ }^{11}$, Jacob S. Ballon (D) ${ }^{1}$, \\ Ruth $\mathrm{O}^{\prime} \mathrm{Hara}^{1,2}$, Douglas F. Levinson (iD) ${ }^{1}$ and Alexander E. Urban (iD) ${ }^{1,9 凶}$
}

This is a U.S. government work and not under copyright protection in the U.S.; foreign copyright protection may apply 2021

\begin{abstract}
Structural variation in the complement 4 gene (C4) confers genetic risk for schizophrenia. The variation includes numbers of the increased C4A copy number, which predicts increased C4A mRNA expression. C4-anaphylatoxin (C4-ana) is a C4 protein fragment released upon $\mathrm{C} 4$ protein activation that has the potential to change the blood-brain barrier (BBB). We hypothesized that elevated plasma levels of C4-ana occur in individuals with schizophrenia (iSCZ). Blood was collected from 15 iSCZ with illness duration $<5$ years and from 14 healthy controls (HC). Plasma C4-ana was measured by radioimmunoassay. Other complement activation products C3-ana, C5-ana, and terminal complement complex (TCC) were also measured. Digital-droplet PCR was used to determine C4 gene structural variation state. Recombinant C4-ana was added to primary brain endothelial cells (BEC) and permeability was measured in vitro. C4-ana concentration was elevated in plasma from iSCZ compared to HC (mean $=654 \pm 16 \mathrm{ng} / \mathrm{mL}, 557 \pm 94$ respectively, $p=0.01$ ). The patients also carried more copies of the $C 4 A L$ gene and demonstrated a positive correlation between plasma C4-ana concentrations and C4A gene copy number. Furthermore, C4-ana increased the permeability of a monolayer of BEC in vitro. Our findings are consistent with a specific role for $C 4 A$ protein in schizophrenia and raise the possibility that its activation product, C4-ana, increases BBB permeability. Exploratory analyses suggest the novel hypothesis that the relationship between C4ana levels and C4A gene copy number could also be altered in iSCZ, suggesting an interaction with unknown genetic and/or environmental risk factors.
\end{abstract}

Translational Psychiatry (2021)11:486; https://doi.org/10.1038/s41398-021-01583-5

\section{INTRODUCTION}

The complement system, a part of the innate immune system, contributes to the first line of defense against pathogens (Fig. 1A). In addition, complement in the brain influences neuronal development and refinement [1]. Dysregulation of complement has been reported in patients with schizophrenia, based on genetic, transcriptomic, and functional studies [2-6].

Genome-wide association studies (GWAS) found an association between schizophrenia and structural variants in the Complement component 4 (C4) gene [7]. C4A and C4B genes have variable gene copy numbers (GCN), and each gene has long (L) and short (S) forms (Fig. 1B). Schizophrenia patients carry more copies of C4A genes and more of the C4AL form, accounting at least in part for the GWAS signal [7]. Mice with higher C4 GCN and increased C4 protein levels show aberrant neuronal development, including increased pruning, accompanied by schizophrenia-like behavioral traits [7-9]. Also linked to schizophrenia is a common intronic allele of the CSMD1 gene [3, 10], which encodes a complement inhibitor expressed in neural tissue, including developing neurons $[11,12]$. CSMD1 deletion in mice leads to neurocognitive deficits [11]. Loss of CSMD1 expression in human stem-cell-derived neurons increases their susceptibility to activated complement, leading to increased complement deposition on neurites [12].

Changes in blood levels of complement proteins also have been reported in schizophrenia [2-4]. A large study of individuals at high risk of schizophrenia found that levels of complement and coagulation proteins and particularly complement pathway inhibitors predicted which individuals subsequently developed psychosis [13]. In addition, the functional (hemolytic) activity of the complement system is consistently higher in individuals with schizophrenia vs. controls [2,3]. Functional or hemolytic activity is measured by exposing patient serum in vitro to antigens that trigger complement cascade activity. The resulting cell lysis provides a measure of how readily the complement system can be triggered. Two small studies found increased C4 hemolytic activity in individuals with schizophrenia compared to controls

\footnotetext{
${ }^{1}$ Department of Psychiatry and Behavioral Sciences, Stanford University School of Medicine, Stanford, CA 94305, USA. ${ }^{2}$ Sierra Pacific Mental Illness Research Education and Clinical Center (MIRECC), VA Palo Alto Health Care System, Palo Alto, CA, USA. ${ }^{3}$ Translational Applications Service Center, Stanford University School of Medicine, Stanford, CA 94305 , USA. ${ }^{4}$ Translational Research and Applied Medicine, Stanford University School of Medicine, Stanford, CA 94305, USA. ${ }^{5}$ Department of Medicine, Stanford University School of Medicine, Stanford, CA 94305, USA. ${ }^{6}$ Department of Psychology, Bar-llan University, Ramat-Gan, Israel. ${ }^{7}$ Department of Neurology, Stanford University School of Medicine, Stanford, CA 94305, USA. ${ }^{8}$ The Neurology Center of Southern California, Temecula, CA 92592, USA. ${ }^{9}$ Department of Genetics, Stanford University School of Medicine, Stanford, CA 94305, USA. ${ }^{10}$ Department of Biomedical Data Science and Statistics, Stanford University School of Medicine, Stanford, CA 94305, USA. ${ }^{11}$ Department of Pediatrics, Stanford Program in Immunology, Stanford University School of Medicine, Stanford, CA 94305, USA. ${ }^{凶}$ email: akalinow@stanford.edu; aeurban@stanford.edu
} 


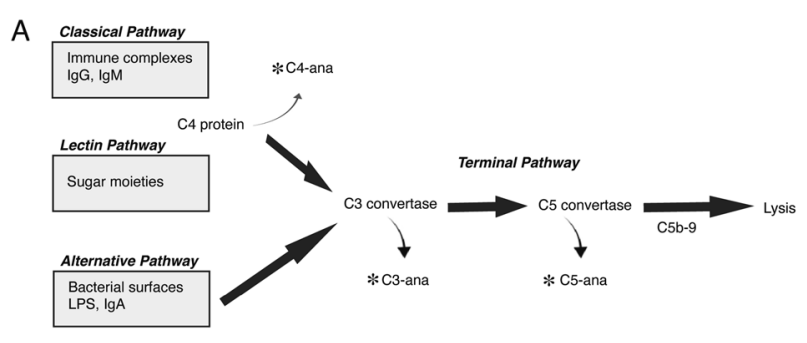

B

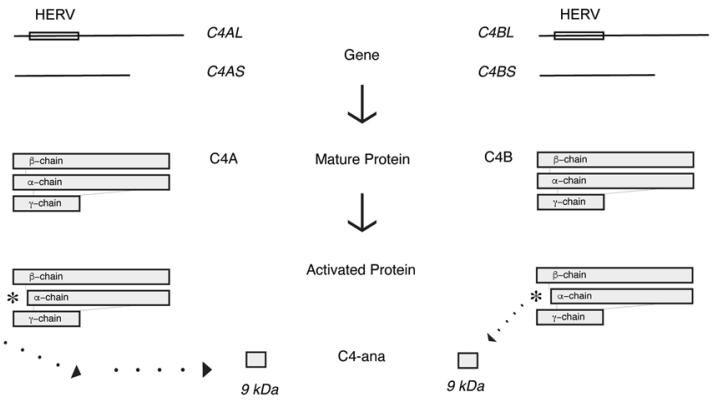

Fig. 1 C4A/B genes produce C4A/B protein, which both release C4-ana when activated. A C4-ana is produced when C4 protein is activated in the Classical and Lectin Complement Pathways. Similarly, C3-ana is released when C3-convertase is activated and C5-ana is produced upon activation of the Terminal Complement Pathway. B An inactive retrovirus, HERV, present between exons 9 and 10 of the C4A or C4B gene, differentiates the long form of the gene from the short form of the gene. The C4A gene produces the C4A (acidic) protein while the C4B gene produces the C4B (basic) protein, differentiated by 5 amino acids as a result of four nucleotide polymorphisms. Both forms of the protein lead to the cleavage product, C4-ana when activated by binding to protein binding partners.

$[14,15]$. An unexplored question is whether variation in C4 structural genotypes contributes to complement activation in the peripheral blood of patients with schizophrenia.

Anaphylatoxins are fragments of complement proteins that are released when the proteins are activated. They are small molecules with half-lives on the order of minutes and have specific downstream receptors and functional effects [16]. C4-ana reflects $\mathrm{C} 4$ protein activation, which occurs in the classical and lectin complement pathways. Protease-activated receptor 1 (PAR1) and protease-activated receptor 4 (PAR4) are the only known C4-ana receptors [17]. C3-ana reflects the activity of C3convertase, which is activated by all three complement cascades. C5-ana is released by the terminal complement cascade. Initiation of any arm of the complement cascade generally results in successive activation of subsequent pathways. The endpoint of the terminal cascade is the formation of a circular protein complex, C5b-9, or terminal complement component (TCC), that embeds itself in cell membranes and causes cell lysis (Fig. 1A). A soluble form of TCC, sTCC, can be measured in plasma and has a half-life $\sim 1 \mathrm{~h}$ [18]. The literature is unclear about which complement pathways are activated in patients with schizophrenia [3]. Measuring activation products provides one way to determine which pathways are active in vivo.

Patients with schizophrenia often have evidence suggesting BBB disruption, including after traumatic brain injury, which increases the risk of schizophrenia $[5,19,20]$. A downstream effect of C4-ana binding to a PAR1/4 receptor is endothelial cell contraction and increased permeability through the endothelial cell layer [17]. Thus, C4-ana activation of PAR1/4 receptors may disrupt the BBB and contribute to neurodegeneration in individuals with schizophrenia. We hypothesized that C4-ana and thus C4 protein are increased in peripheral blood early in schizophrenia. We carried out an initial test of this hypothesis in individuals with schizophrenia with an illness duration of less than five years vs. control individuals with similar age, sex, and ethnicity.

\section{MATERIALS AND METHODS}

\section{Recruitment of participants}

We recruited 15 individuals with schizophrenia or schizoaffective disorder from academic inpatient and outpatient settings. Participants were ages 18-35, unrestricted with regard to ethnicity. Additional inclusion criteria for participants with schizophrenia included a clinical DSM-V diagnosis of schizophrenia or schizoaffective disorder, confirmation of one of these diagnoses by the Structured Clinical Interview for DSM-V (SCID-V), and initial diagnosis or initiation of antipsychotic medication within the last 5 years. Exclusion criteria included a positive urine toxicology screen or selfreported history of any of the following: current substance abuse or any use of cannabis or tobacco products, a history of bleeding disorders, excessive bleeding with previous surgery, taking blood thinners, autoimmune conditions, epilepsy, known genetic disorders, immunocompromised state, pregnancy, history of central nervous system disease, an uncontrolled medical disorder such as cancer or inability to provide informed consent. The 14 healthy control participants were matched to the same age range as the group of individuals with schizophrenia, did not meet criteria for any DSM- 5 disorder by the SCID-V, and had a Distress Score of less than or equal to 6 on the Prodromal Questionnaire-Brief Version (PQ-B) [21].

\section{Clinical assessment}

Clinical evaluation was carried out by trained doctoral-level clinical research interviewers. Participants were assessed for their capacity to participate in the research study upon interview and according to best practices [22]. All participants underwent a SCID-V interview. Controls also completed a PQ-B and patients completed a Positive and Negative Symptom Score Structured Interview (PANSS). Participants were asked about current medications (see Supplementary Material for details). Participants who completed the clinical evaluation and venipuncture were invited to return for a subsequent visit for an optional lumbar puncture.

The study protocol was approved by the Stanford University Institutional Review Board (IRB Protocol 47825). All participants gave written informed consent. Participants were compensated for study participation.

\section{Sample collection}

All participants underwent antecubital venipuncture between 08:00 AM and 12:00 PM at the Clinical Translational Research Unit (CTRU). Blood was collected in EDTA tubes and transported on ice to the Stanford Translational Applications Service Center (TASC) for centrifugation at $1000 \times g$ for $10 \mathrm{~min}$ at $4^{\circ} \mathrm{C}$ within $30 \mathrm{~min}$ of collection. Immediate cooling halts protein metabolism since enzymes are optimally active at body temperature, $37^{\circ} \mathrm{C}$. The supernatant was aliquoted and snap-frozen in liquid nitrogen and stored at $-80^{\circ} \mathrm{C}$ until samples were analyzed. Participants underwent height and weight measurements at the time of blood draw. All collected samples were used in the analyses.

\section{Complement activation product measurements}

Samples were shipped overnight on dry ice to Complement Laboratory of the National Jewish Medical Center (Denver, CO) where they underwent radioimmunoassay measurement of plasma and CSF C3-ana, C4-ana, and C5-ana concentrations in a CLIA-certified laboratory. STCC, or soluble terminal complement product, concentration was measured according to manufacturer's instructions using the Human Terminal Complement Complex ELISA kit (HK328 Hycult Biotech, Netherlands) in technical duplicates and averaged per sample.

\section{C4 genotype copy number determination}

DNA was extracted from 0.5 to $1 \mathrm{~mL}$ frozen whole blood collected in EDTA tubes using the DNAeasy Blood \& Tissue Kit (Qiagen, Netherlands). C4A and $\mathrm{C} 4 \mathrm{~B}$ structural polymorphism genotypes were assayed with digitaldroplet PCR (ddPCR; BioRad, Pleasanton, CA) with the method of Sekar 
Table 1. Demographics of study participants.

\begin{tabular}{llll} 
& Controls & Schizophrenia & p-value \\
\hline \# Participants & 14 & 15 & \\
\hline Median age & 24 & 22 & 0.14 \\
\hline Age range & $21-36$ & $18-34$ & \\
\hline Sex (\% male) & $64 \%$ & $73 \%$ & 0.46 \\
\hline Caucasian & 9 & 5 & \\
\hline African American & 2 & 2 & \\
\hline Asian & 3 & 5 & \\
\hline Hispanic & 1 & 2 & \\
\hline Schizophrenia & 0 & 12 & 0.01 \\
\hline Schizoaffective disorder & 0 & 3 & \\
\hline BMl, average & 24 & 27.5 &
\end{tabular}

There were no statistical differences in participant age or sex between the two groups. The average BMI (body mass index) was higher in the Schizophrenia group. Attempts were made to recruit participants from a variety of ethnic backgrounds. The majority of participants in the Schizophrenia group met the criteria for the diagnosis of Schizophrenia and 3 met DSM-V criteria for Schizoaffective disorder.

et al. [7]. Briefly, in this assay PCR probes for C4A, C4B, C4L and C4S are assayed as technical duplicates. A long-range PCR for $C 4 S$ is then performed whose products are analyzed by ddPCR to determine the Gene Copy Numbers (GCN) of C4AS and C4BS, following which GCN can be computed for C4AL and C4BL. Predicted C4A and C4B expressions are then determined according to a formula [7] derived from post-mortem genotype and brain expression data:

C4A expression $=(0.47 *$ C4AL GCN $)+(0.47 *$ C4AS GCN $)+(0.20 *$ (4BL GCN)

C4B expression $=(1.03 *$ C4BL GCN $)+(0.88 *$ C4BS GCN $)$

\section{Statistical analysis}

The primary outcome was plasma C4-ana concentration in the control vs. patient groups. All other statistical tests were considered exploratory and thus were not corrected for the number of tests. Protein concentrations (C3-, C4- and C5-ana, and STCC) and C4 GCN frequencies were not normally distributed (Shapiro-Wilk test) so that nonparametric (rank-based) tests were used for group contrasts (Mann-Whitney U-Test and correlational analyses Spearman's rho). Differences between pairs of rho values were tested for significance using the appropriate modification of Fisher's Z-transformation of correlation coefficients $[23,24]$. All analyses were performed using JMP Pro 14.1.0 (SAS Institute, Cary, NC).

\section{Primary brain endothelial cell (BEC) culture}

Primary human brain microvascular endothelial cells (ACBRI 376, Cell Systems, Kirkland, WA) were cultured in Complete Classic Medium with Serum and CultureBoost (4Z0-500, Cell Systems, Kirkland WA) under standard conditions $\left(37^{\circ} \mathrm{C}\right.$ and $\left.5 \% \mathrm{CO}_{2}\right)$ until a monolayer was formed. Attachment Factor (4Z0-210, Cell Systems, Kirkland, WA) and Passage Reagent Group (4Z0-800, Cell Systems, Kirkland, WA) were used for passaging according to the manufacturer's instructions. Cells were tested for mycoplasma.

\section{Immunofluorescence}

Primary BEC were cultured on glass chamber slides (80827, ibidi $\mathrm{GmbH}$, Germany). Cells were starved in media without serum and exposed to stimulants for $30 \mathrm{~min}$. Then, cells were fixed in $4 \%$ paraformaldehyde in PBS pH 7.4 for $10 \mathrm{~min}$ at room temperature and washed $3 \times$ with ice-cold PBS for 5 min. Cells were permeabilized with $0.1 \%$ Triton X-100 in PBS for $10 \mathrm{~min}$ and stained with Texas Red-X Phalloidin for $20 \mathrm{~min}$ at room temperature (T7471, ThermoFischer Scientific, Waltham, MA) or alternatively incubated overnight at $4{ }^{\circ} \mathrm{C}$ with primary antibody against PAR1 (sc-13503, Santa Cruz Biotechnology Inc., Dallas, TX) and PAR4 (sc1666, Santa Cruz Biotechnology Inc., Dallas, TX). The samples were subsequently washed and incubated with secondary antibody (A21203, Life Technologies, Eugene OR) for $1 \mathrm{~h}$ at room temperature, and $5 \mathrm{~min}$ with DAPI (Sigma Aldrich, St. Louis, MO). Cells were imaged at the Stanford University Cell Sciences Imaging Facility on a Zeiss 880 confocal microscope using a $\times 40$ objective.

\section{In vitro permeability assay}

Transwell plates (Corning, Corning NY) were coated overnight at $4{ }^{\circ} \mathrm{C}$ with $10 \mu \mathrm{g} / \mathrm{mL}$ of rat tail collagen (Corning, Corning NY). Then, cells were grown on the transwell inserts until a monolayer formed. Stimulants, thrombin (T4393, Sigma Aldrich, MO) or C4-anaphylatoxin (A106, Complement Technologies, Tyler, TX) were added for $1 \mathrm{~h}$, and permeability was measured according to manufacturer's instructions (CB6929, Cell Biologics, Chicago, IL). The experiment was performed three times, each with technical duplicate wells per experimental condition.

\section{RESULTS}

\section{Characteristics of the cohort}

A total of 19 controls and 16 individuals with schizophrenia enrolled in the study. Four individuals in the control group were ineligible after SCID-V assessment, and 1 control and 1 individual with schizophrenia withdrew before completing the study. Thus, 14 healthy controls and 15 participants with schizophrenia or schizoaffective disorder completed the clinical evaluation and blood sampling (Table 1). The two groups did not differ significantly with respect to age, sex distribution, or ethnicity. Together, the individuals with schizophrenia had a higher body mass index (BMI). All of the individuals with schizophrenia except one (in remission) were taking medications at the time of venipuncture (Supplementary Fig. 1). The C4-ana concentration of the medication-free individual with schizophrenia was in the upper range $(688 \mathrm{ng} / \mathrm{mL})$.

\section{Plasma C4-ana concentration}

Concentrations of plasma C4-ana were elevated in individuals with schizophrenia compared to controls (mean \pm standard deviation $=656 \pm 150 \mathrm{ng} / \mathrm{mL}$ vs. $555 \pm 93 \mathrm{ng} / \mathrm{mL}, X^{2}$ approximation $[1 \mathrm{df}]=5.97, p$-value $=0.015$ ) (Fig. 1B). This difference persisted after excluding the individual with schizophrenia whose C4-ana concentration $(1142 \mathrm{ng} / \mathrm{mL})$ was an outlier value $(620 \pm$ $68 \mathrm{ng} / \mathrm{mL}$ vs. $555 \pm 93 \mathrm{ng} / \mathrm{mL}, X^{2}$ approximation [1df] $=5.07, p$ value $=0.024$ ). There are no clear relationships between plasma C4-ana concentration and BMI, fasting status or sex (Supplementary Fig. 2).

\section{Additional complement split products in plasma}

Results are shown in Fig. 2. There is a trend towards increased C3-ana concentrations in individuals with schizophrenia $(189 \pm 37 \mathrm{ng} / \mathrm{mL}$ vs. $170 \pm 19 \mathrm{ng} / \mathrm{mL}, x^{2}$ approximation [1df] = 2.90, $p$-value $\left.=0.09\right)$. C5ana and TCC concentrations did not differ between groups.

\section{Complement split products in CSF}

Only 5 patients and 6 controls completed lumbar puncture. Procedures are discussed and preliminary data are shown in Supplementary Fig. 3.

\section{C4 genotype distribution}

Figure $3 \mathrm{~A}$ shows the distributions of $\mathrm{C} 4$ gene copy numbers (GCN) in individuals with schizophrenia and controls for the individual alleles (C4AL, C4AS, C4BL, C4BS) and for aggregate alleles (C4A, $\mathrm{C} 4 \mathrm{~B}$, and $\mathrm{C4}$ ). Exploratory analyses showed an increase in $\mathrm{C} 4 \mathrm{AL}$ $(p=0.03)$ in individuals with schizophrenia and a trend for all C4A $(p=0.06)$ (Fig. 3B). 
A

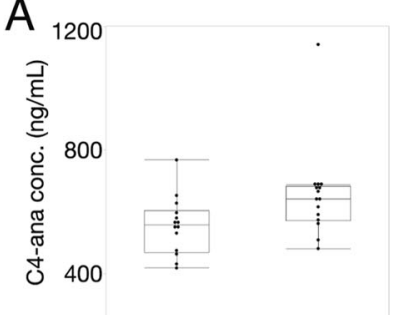

Controls Schizophrenia

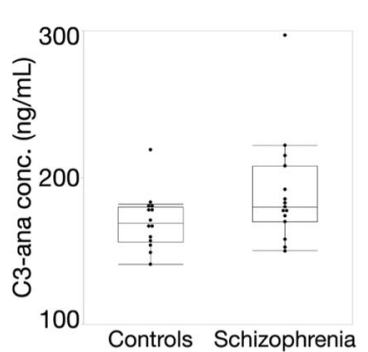

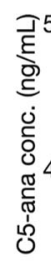

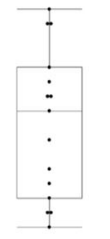

Controls Schizophrenia

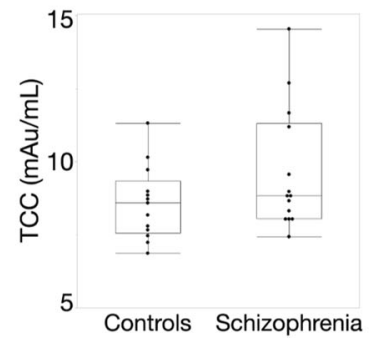

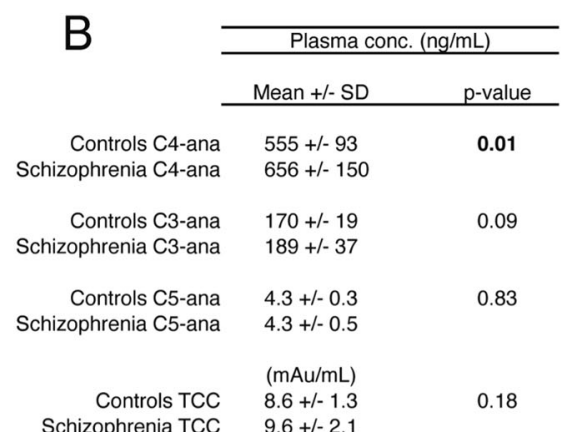

Schizophrenia TCC $\quad 9.6+/-2.1$

Fig. 2 C4-ana concentration is higher in plasma from individuals with schizophrenia. A Concentration of C4-ana, C3-ana, and C5-ana in plasma in the whole cohort. The mean concentration of C4-ana is higher for the patient group compared to controls, $p=0.01$ ( $p=0.02$ if the outlier is removed), as calculated by Mann-Whitney. The mean plasma levels of C3-ana, C5-ana are lower in the control group when compared to the group from individuals with schizophrenia. Detailed values and statistics are shown in the table in $\mathbf{B}$.

\section{The relationship between $\mathrm{C} 4$ gene copy number and plasma C4-ana levels}

Figure $3 C$ shows the correlations between plasma $C 4$-ana and (i) GCN values (C4, C4A, C4B, C4AL, C4AS, C4BL, C4BS); and (ii) predicted $C 4 A$ and $C 4 B$ gene expression in the brain. In the entire cohort, the only nominally significant correlation was an inverse relationship between plasma $C 4$-ana and predicted $C 4 \mathrm{~B}$ expression $(\rho=-0.37, p=0.05)$. We then analyzed individuals with schizophrenia and controls separately. In individuals with schizophrenia, plasma C4-ana was positively correlated with C4A GCN $(\rho=0.69, p=0.005)$; in controls, there was no correlation $(\rho=-0.69, p=0.15)$. The correlations in individuals with schizophrenia and in controls were significantly different based on the modified Fisher's Z-transformation test for rho $(z$ [difference $]=-2.371, p=0.018)$ (Fig. 3D). This difference was sensitive to the removal of the patient outlier $(p=0.61$ in the patients, $N=14 ; z$ [difference] $=-1.79, p=0.073$ ). The groupspecific correlations between C4-ana and C4B GCN were in the opposite directions and were not significantly different from each other (cases: $p=-0.68, p=0.006$; controls: $p=0.24, p=$ $0.41 ; z$ [difference] $=1.11, p=0.27)$. A negative correlation between C4A and C4B GCNs is expected, based on population allele frequencies [7], as observed also in C4 GCN assays in European-, African- and Chinese-ancestry individuals [DF Levinson, unpublished data].

\section{C4-ana increases in vitro permeability of brain endothelial cells in monolayer cultures}

We hypothesized that increased C4-ana could have a pathological effect by binding to PAR1 and PAR4 [17] on brain endothelial cells (BEC) and causing increased permeability. We tested this hypothesis by studying monolayer BEC cultures. We confirmed that PAR1 and PAR4 receptors are present on BEC (Supplementary Fig. 4) and observed that exposure to C4-ana increased actin stress fiber formation (Fig. 4A). C4-ana, like thrombin, increased permeability of the BEC monolayer. In this assay, C4-ana was $\sim 10 \times$ less potent than thrombin (Fig. 4B).

\section{DISCUSSION}

Elevated C4 gene copy number has been implicated in schizophrenia etiology by GWAS [7] and by murine models [7-9]. An allele of the complement inhibitor, CSMD1, is also associated with schizophrenia [3, 11, 12]. In addition, peripheral inflammation may contribute to disease pathogenesis through changes to the BBB [25]. Based on these considerations and our pilot data from individuals with schizophrenia, we hypothesized that circulating complement fragments, known to be inflammatory mediators, could be increased in this disease. To address this hypothesis, we measured the concentrations of anaphylatoxins (C3-ana, C4-ana, C5-ana) and TCC from carefully collected plasma, which provides a way to determine complement protein activation in vivo, rather than ex vivo methods that trigger activation [18].

We found significantly higher plasma C4-ana levels, but not C3ana or C5-ana levels, in individuals with schizophrenia compared to healthy controls (Fig. 2). A previous small study of drug-naive, first-episode psychosis patients measured their TCC, C3-, and C5ana (but not C4-ana) levels and found a trend toward elevated C3ana. Notably, this trend was eliminated after treatment [26]. The latter result is consistent with our findings, as the majority of our participants were taking medication at the time of sampling. Two prior studies have measured in vitro activation of C4 protein, isolated from individuals with schizophrenia and controls [14, 15]. In both studies, C4 activation from individuals with schizophrenia was enhanced. This is also consistent with our results, as C4-ana is an in vivo by-product of C4 protein activation. Furthermore, several studies observed increased levels of a C4 protein inhibitor, C4-binding protein, in individuals with schizophrenia, implying an active compensatory mechanism $[13,27]$. Together, these findings suggest that plasma C3-ana may play a role in acute psychosis, whereas plasma C4-ana may contribute in a more chronic manner. In general, there are no known disease associations for C4-ana, we found just one small study that showed elevated concentrations of C4-ana in multiple sclerosis [28].

Consistent with prior work [7], we found significantly increased C4AL GNC and a trend towards increased total C4A GCN in individuals with schizophrenia compared to controls (Fig. 3). 
A
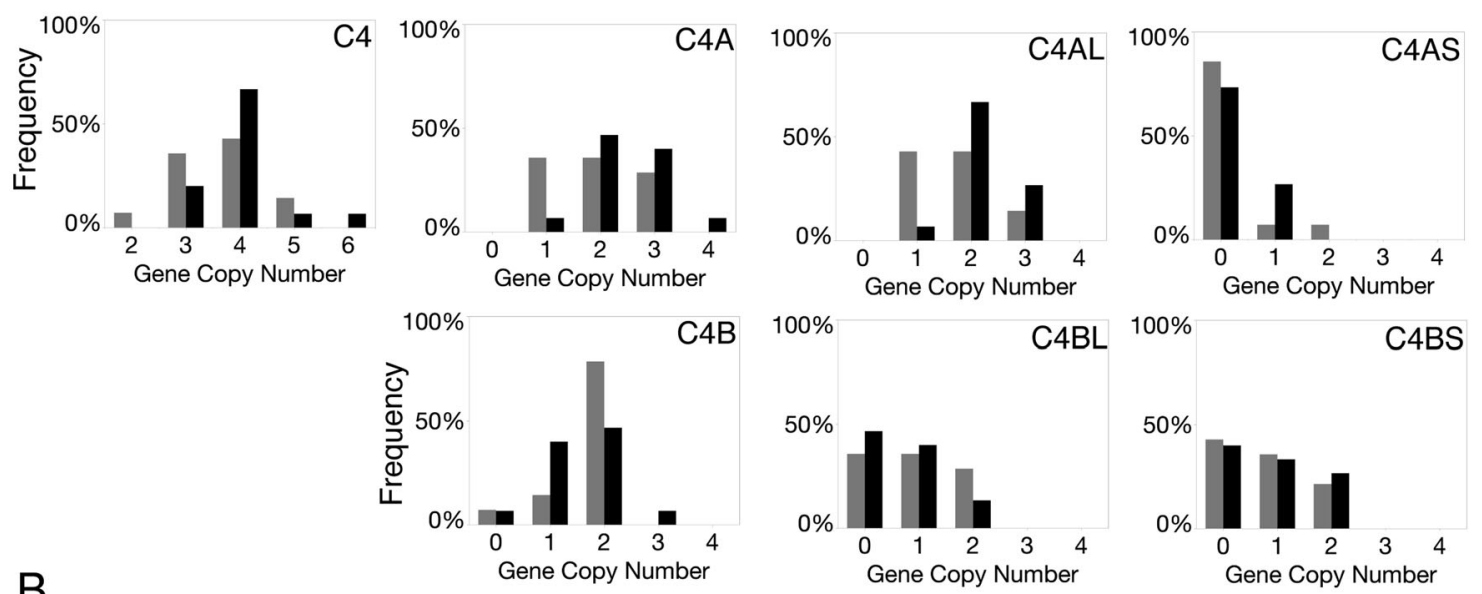

B

\begin{tabular}{|c|c|c|c|}
\hline Gene copy number & Controls & Schizophrenia & Mann-Whitney \\
\hline & Mean +/-SD & Mean +1-SD & $p$-value \\
\hline Total C4 & $3.6+/-0.83$ & $4.0+/-0.76$ & 0.2 \\
\hline Total C4A & $1.9+/-0.83$ & $2.5+/-0.74$ & 0.06 \\
\hline Total C4B & $1.7+/-0.59$ & $1.5+/-0.74$ & 0.28 \\
\hline C4AL & $1.7+/-0.72$ & $2.2+/-0.56$ & 0.03 \\
\hline C4AS & $0.2+1-0.56$ & $0.27+1-0.46$ & 0.46 \\
\hline C4BL & $0.87+/-0.83$ & $0.67+/-0.72$ & 0.52 \\
\hline C4BS & $0.86+/-0.83$ & $0.86+1-0.83$ & 1 \\
\hline
\end{tabular}
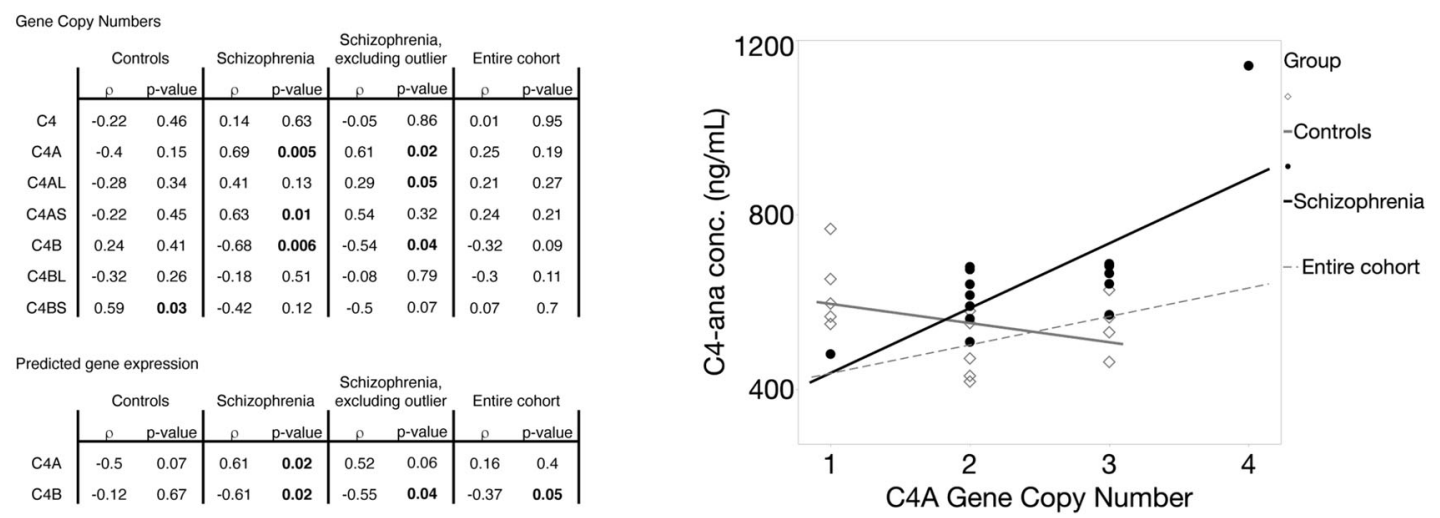

Fig. 3 The relationship between C4-ana concentration and $\mathbf{C} 4$ gene copy number differs in cases vs. controls. A The number of C4AL, C4AS, C4BL, and C4BS genes were determined experimentally by ddPCR. The frequency of each gene variant in the control and individuals with schizophrenia groups are reported. Total C4A was determined by adding the number of C4AL and C4AS gene copies for each participant. Similarly, C4B was determined by adding the number of C4BL and C4BS gene copies for each participant. Total C4 was determined by adding the total gene copies of $C 4 A$ and $C 4 B$ for each participant. The frequencies of each gene copy number for participants are reported in the graphs above. The average gene copy numbers for each distribution are reported in table (B). In this cohort, we see that samples from individuals with schizophrenia have higher gene copy numbers of total C4A and C4AL genes compared to controls ( $p$-value $=0.04$ and 0.03 , respectively. These $p$-values are not statistically significant if the Bonteferoni correction is applied). C The table shows results of Spearman's rho correlation analyses between plasma C4-ana concentration and (i) each subset of C4 gene copy numbers (total C4, C4A, C4B, C4AL, C4AS, C4BL, C4BS); and (ii) predicted C4A and C4B gene expression in brain based on the formula of Sekar et al. D Plasma C4-ana concentration ( $Y$ axis) is plotted against total C4A ( $X$ axis), with regression lines shown for the entire cohort and separately for cases and controls. Cases showed a positive relationship, and controls an inverse relationship, between C4A GCN and plasma C4A GCN.

Studies of post-mortem brain tissue showed higher C4A mRNA levels in individuals with schizophrenia compared to controls [5-7]. One study of peripheral blood mononuclear cell transcripts found increased C4A mRNA that correlated with PANSS positive symptoms factor $p$-scores, driven by the severity of delusions [29]. However, in two other studies, C4 mRNA levels in peripheral blood were not increased $[6,30]$. In healthy individuals, peripheral blood
$\mathrm{C} 4 \mathrm{~A}$ and $\mathrm{C} 4 \mathrm{~B}$ protein concentrations correlate with the $\mathrm{C} 4 \mathrm{~A}$ and $C 4 B$ gene copy number but also depend on age and sex [31, 32]. The literature on the serological complement system in schizophrenia is large but characterized by small samples, variable methodological approaches, clinical diversity of cohorts, failure to assay $C 4 A$ and $C 4 B$ separately in most studies, and inconsistent results $[2,3,27]$. A recent meta-analysis of 10 studies of $C 4$ protein 

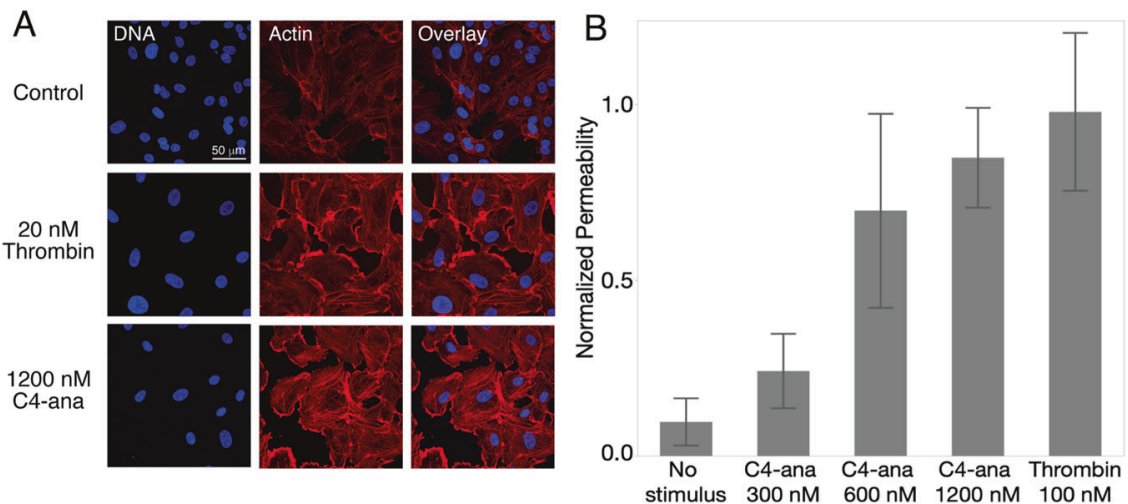

Fig. 4 C4-ana increases the permeability of primary brain endothelial cells. A Brain endothelial cells stained with phalloidin and DAPI. After BEC are exposed to C4-ana, cells form actin stress fibers. B The permeability of the monolayer increases after exposure to increasing concentrations of C4-ana and thrombin.

concentration (mostly in serum) included 468 individuals with schizophrenia and 440 controls. This study did not find a difference between individuals with schizophrenia and controls, but did note a larger range of concentrations in the individuals with schizophrenia (23). Importantly, the liver is the major source of plasma C4 [33], but no studies have examined C4 mRNA in the liver in individuals with schizophrenia.

We also found a positive, significant correlation between plasma C4-ana and C4A GCN in individuals with schizophrenia (Fig. 3). A possible explanation is an enhanced susceptibility to activation of $\mathrm{C} 4$ protein in individuals with schizophrenia, discussed above. A mechanism for the enhancement could involve the effects of interferon- $\gamma$ (INF- $\gamma$ ), a pro-inflammatory cytokine, that is the strongest known inducer of C4 protein expression $[33,34]$. A recent meta-analysis found INF- $\gamma$ to be elevated in both individuals with schizophrenia and first-episode psychosis (FEP) [35]. In a study of FEP patients who went on to a diagnosis of schizophrenia, INF- $\gamma$ was elevated in FEP compared to controls and inversely correlated with the percentage of whole-brain gray matter [36]. INF- $\gamma$ induction of C4 protein expression would result in more C4A-type protein in individuals with schizophrenia because they carry more copies of the C4A gene. C4A protein has differential biochemical properties and functions in synaptic pruning $[9,37]$ and may also be more susceptible to activation. Examining the correlation between INF$\gamma$ and C4-ana concentrations as a function of C4 GCN in both individuals with schizophrenia and controls would begin to test this hypothesis. Alternatively, unknown factors may affect C4 protein activation.

Functions of C3-ana and C5-ana are well-characterized. They bind to $\mathrm{G}$ protein-coupled receptors, $\mathrm{C} 3 \mathrm{aR}$ and $\mathrm{C} 5 \mathrm{aR} 1$, respectively, induce migration and effector function in neutrophils, mast cells, and macrophages and increase the permeability of small blood vessels [38]. In contrast, surprisingly little is known about the biology of C4-ana [39]. Although named an "anaphylatoxin," C4-ana does not have the same mechanisms of action as C3-ana and C5-ana. The C4-ana receptor, PAR1, is expressed on neurons and astrocytes $[40,41]$. Activation of PAR1 leads to apoptotic cell death in neurons [41], and to glutamate release in astrocytes producing activation of neighboring NMDA receptors on neurons [41]. These mechanisms are consistent with the glutamatergic excess found in individuals with and at risk for schizophrenia [42].

PAR1 is also expressed on leukocytes, platelets, and endothelial cells (16), including BEC [43]. Indeed, the only well-established downstream effect of C4-ana binding to a PAR1/4 receptor is endothelial cell contraction and increased permeability through the endothelial cell layer [17].

We found that the addition of C4-ana increases the permeability of a BEC monolayer (Fig. 4). A recent in vitro study demonstrated that PAR1 activation is required for lymphocyte transmigration in BEC [44]. There is evidence that suggests BBB disruption in individuals with schizophrenia $[5,19,20]$. Thus, C4-ana activation of PAR1/4 receptors may be a contributing mechanism to changing the $\mathrm{BBB}$ and contribute to neurodegeneration in individuals with schizophrenia.

The limitations of this study include its small sample size which limited our ability to measure group differences of several activation products. Future studies where larger sample sizes and additional makers of protein activation are needed. We were also unable to exclude two possible confounders: (i) the effect of medication and (ii) BMI on complement activation products. (i) One previous study reported an increase in C3-ana after medication treatment [26], however other studies examining the complement system did not find changes related to medication $[2,3]$. (ii) We observed a trend toward a correlation between BMI and C4-ana concentrations, but only in the patient group (Supplemental Fig. 1). One study reported that BMI was strongly correlated with serum $C 3$ and weakly with serum $C 4 A$ and $C 4 B$ concentrations [31, 45]. A second study reported that metabolic syndrome and waist circumference were associated with total serum C4 at baseline in a longitudinal study, with C4 level predicting the incidence of metabolic syndrome over time (C4-ana was not assayed) [31, 45]. C4-ana has not previously been studied in relation to $\mathrm{BMI}$ or related variables. Our data do not permit disentangling the relationships among C4-ana, antipsychotic medication, and BMI.

Further work is needed to test hypotheses arising from the current findings: that a peripheral mechanism of C4 protein activation in patients with schizophrenia interacts with increased C4 GNC and structural variants, leading to increased C4-ana. Given the small sample size of our study, larger cohorts are needed in FEP and individuals with chronic schizophrenia.

\section{REFERENCES}

1. Gorelik A, Sapir T, Haffner-Krausz R, Olender T, Woodruff TM, Reiner O. Developmental activities of the complement pathway in migrating neurons. Nat Commun. 2017;8:1-12

2. Mayilyan KR, Weinberger DR, Sim RB. The complement system in schizophrenia. Drug News Perspect. 2008;21:200-10.

3. Woo JJ, Pouget JG, Zai CC, Kennedy JL. The complement system in schizophrenia: where are we now and what's next? Mol Psychiatry. 2020;25:114-30. 
4. Magdalon J, Mansur F, Teles E Silva AL, de Goes VA, Reiner O, Sertié AL. Complement system in brain architecture and neurodevelopmental disorders. Front Neurosci. 2020;14:1-14

5. Gandal MJ, Zhang P, Hadjimichael E, Walker RL, Chen C, Liu S, et al. Transcriptome-wide isoform-level dysregulation in ASD, schizophrenia, and bipolar disorder. Science. 2018;362:eaat8127.

6. Rey R, Suaud-Chagny MF, Bohec AL, Dorey JM, d'Amato T, Tamouza R, et al. Overexpression of complement component $\mathrm{C} 4$ in the dorsolateral prefrontal cortex, parietal cortex, superior temporal gyrus and associative striatum of patients with schizophrenia. Brain Behav Immun. 2020;90:216-25.

7. Sekar A, Bialas AR, de Rivera H, Davis A, Hammond TR, Kamitaki N, et al. Schizophrenia risk from complex variation of complement component 4. Nature. 2016;530:177-83.

8. Comer AL, Jinadasa T, Sriram B, Phadke RA, Kretsge LN, Nguyen TPH, et al. Increased expression of schizophrenia-associated gene $\mathrm{C} 4$ leads to hypoconnectivity of prefrontal cortex and reduced social interaction. PLoS Biol. 2020;18:e3000604.

9. Yilmaz M, Yalcin E, Presumey J, Aw E, Ma M, Whelan CW, et al. Overexpression of schizophrenia susceptibility factor human complement C4A promotes excessive synaptic loss and behavioral changes in mice. Nat Neurosci. 2020;24:214-24. https://doi.org/10.1038/s41593-020-00763-8.

10. Rivas MA, Beaudoin M, Gardet A, Stevens C, Sharma Y, Zhang CK, et al. Genome-wide association study identifies five new schizophrenia loci. Nat Genet. 2011;43:969-78.

11. Steen VM, Nepal C, Ersland KM, Holdhus R, Nævidal M, Ratvik SM, et al. Neuropsychological deficits in mice depleted of the schizophrenia susceptibility gene CSMD1. PLOS ONE. 2013:8:79501.

12. Baum ML, Wilton DK, Muthukumar A, Fox RG, Carey A, Crotty W, et al. CUB and sushi multiple domains 1 (CSMD1) opposes the complement cascade in neural tissues. bioRxiv. 2020;1:1-56.

13. Mongan D, Föcking M, Healy C, Susai SR, Heurich M, Wynne K, et al. Development of proteomic prediction models for transition to psychotic disorder in the clinical high-risk state and psychotic experiences in adolescence. JAMA Psychiatry. 2020;78:77-90. https://doi.org/10.1001/jamapsychiatry.2020.2459.

14. Hakobyan S, Boyajyan A, Sim RB. Classical pathway complement activity in schizophrenia. Neurosci Lett. 2005;374:35-37.

15. Mayilyan KR, Arnold JN, Presanis JS, Soghoyan AF, Sim RB. Increased complement classical and mannan-binding lectin pathway activities in schizophrenia. Neurosci Lett. 2006;404:336-41.

16. Merle NS, Church SE, Fremeaux-Bacchi V, Roumenina LT. Complement system part I - molecular mechanisms of activation and regulation. Front Immunol. 2015;6:1-30.

17. Wang HBin, Ricklin D, Lambris JD. Complement-activation fragment C4a mediates effector functions by binding as untethered agonist to protease-activated receptors 1 and 4. Proc Natl Acad Sci USA. 2017;114:10948-53.

18. Bergseth G, Ludviksen JK, Kirschfink M, Giclas PC, Nilsson B, Mollnes TE. An international serum standard for application in assays to detect human complement activation products. Mol Immunol. 2013;56:232-9.

19. Orsini F, De Blasio D, Zangari R, Zanier ER, De Simoni MG. Versatility of the complement system in neuroinflammation, neurodegeneration and brain homeostasis. Front Cell Neurosci. 2014;8:1-19.

20. Pollak TA, Drndarski $S$, Stone JM, David AS, McGuire $P$, Abbott NJ. The blood-brain barrier in psychosis. Lancet Psychiatry. 2018;5:79-92.

21. Loewy RL, Pearson R, Vinogradov S, Bearden CE, Cannon TD. Psychosis risk screening with the Prodromal Questionnaire - Brief Version (PQ-B). Schizophr Res. 2011;129:42-46.

22. Nishimura AJ, Erwin PJ, Tilburt JC, Murad MH, McCormick JB. Improving understanding in the research informed consent process: a systematic review of 54 interventions tested in randomized control trials. BMC Med Ethics. 2013;14:28.

23. Sheskin D. Handbook of parametric and nonparametric statistical procedures. Boca Raton: Chapman \& Hall/CRC; 2004.

24. Zarr J. Biostatistical analysis. Upper Saddle River: Prentice Hall; 1999.

25. Kealy J, Greene C, Campbell M. Blood-brain barrier regulation in psychiatric disorders. Neurosci Lett. 2020;726:133664.

26. Kucharska-Mazur J, Tarnowski M, Dołęgowska B, Budkowska M, Pędziwiatr D, Jabłoński $M$, et al. Novel evidence for enhanced stem cell trafficking in antipsychotic-naïve subjects during their first psychotic episode. J Psychiatr Res. 2014;49:18-24.

27. Mongan D, Sabherwal S, Susai SR, Föcking M, Cannon M, Cotter DR. Peripheral complement proteins in schizophrenia: A systematic review and meta-analysis of serological studies. Schizophr Res. 2020;222:58-72.

28. Wild G, Watkins J, Ward AM, Hughes P, Hume A, Rowell NR. C4a anaphylotoxin levels as an indicator of disease activity in systemic lupus erythematosus. Clin Exp Immunol. 1990;80:167-70.
29. Melbourne JK, Rosen C, Feiner B, Sharma RP. C4A mRNA expression in PBMCs predicts the presence and severity of delusions in schizophrenia and bipolar disorder with psychosis. Schizophr Res. 2018;197:321-7.

30. zhang T, Tang Y, Yang X, Wang X, Ding S, Huang K, et al. Expression of GSK3 3 , PICK1, NEFL, C4, NKCC1 and Synaptophysin in peripheral blood mononuclear cells of the first-episode schizophrenia patients. Asian J Psychiatr. 2021;55:102520.

31. Yang Y, Chung EK, Zhou B, Blanchong CA, Yu CY, Füst G, et al. Diversity in intrinsic strengths of the human complement system: serum $\mathrm{C} 4$ protein concentrations correlate with C4 gene size and polygenic variations, hemolytic activities, and body mass index. J Immunol. 2003;171:2734-45.

32. Kamitaki N, Sekar A, Handsaker RE, de Rivera H, Tooley K, Morris DL, et al Complement genes contribute sex-biased vulnerability in diverse disorders. Nature. 2020;582:577-81.

33. Kilicarslan A, Uysal A, Charles E. Acute phase reactants. Acta Med Cordoba. 2013;2:2-7.

34. Vastag M, Skopál J, Kramer J, Kolev K, Vokó Z, Csonka E, et al. Endothelial cells cultured from human brain microvessels produce complement proteins factor $\mathrm{H}$, factor B, C1 inhibitor, and C4. Immunobiology. 1998;199:5-13.

35. Goldsmith DR, Rapaport MH, Miller BJ. A meta-analysis of blood cytokine network alterations in psychiatric patients: comparisons between schizophrenia, bipolar disorder and depression. Mol Psychiatry. 2016;21:1696-709.

36. Lesh TA, Careaga M, Rose DR, McAllister AK, Van de Water J, Carter CS, et al Cytokine alterations in first-episode schizophrenia and bipolar disorder: relationships to brain structure and symptoms. J Neuroinflammation. 2018;15:1-11.

37. Isenman DE, Young JR. Covalent binding properties of the C4A and C4B isotypes of the fourth component of human complement on several C1-bearing cell surfaces. J Immunol. 1986;136:2542-25450.

38. West $\mathrm{EE}$, Kolev $\mathrm{M}$, Kemper $\mathrm{C}$. Complement and the regulation of $\mathrm{T}$ cell responses. Annu Rev Immunol. 2018;36:309-38.

39. Barnum SR. C4a: an anaphylatoxin in name only. J Innate Immun. 2015;7:333-9. 40. Junge CE, Lee CJ, Hubbard KB, Zhang Z, Olson JJ, Hepler JR, et al. Proteaseactivated receptor-1 in human brain: Localization and functional expression in astrocytes. Exp Neurol. 2004;188:94-103.

41. Luo W, Wang Y, Reiser G. Protease-activated receptors in the brain: Receptor expression, activation, and functions in neurodegeneration and neuroprotection. Brain Res Rev. 2007;56:331-45.

42. Plitman E, Nakajima S, Fuente-sandoval CDL, Gerretsen $P$, Chakravarty $M M$ Kobylianskii J, et al. Glutamate-mediated excitotoxicity in schizophrenia: a review. Eur Neuropsychopharmacol. 2015;24:1591-605.

43. Kim YV, Di Cello F, Hillaire CS, Kim KS. Differential Ca2+ signaling by thrombin and protease-activated receptor-1-activating peptide in human brain microvascular endothelial cells. Am J Physiol. 2004;286:C31-42.

44. Dragoni S, Papageorgiou A, Araiz C, Greenwood J, Turowski P. Signalling is required for lymphocyte transmigration across brain microvascular. Cells. 2020;9:1-18.

45. Xin Y, Hertle E, van der Kallen C, Schalkwijk CG, Stehouwer C, van Greevenbroek M. Complement $\mathrm{C} 3$ and $\mathrm{C} 4$, but not their regulators or activated products, are associated with incident metabolic syndrome: the CODAM study. Endocrine. 2018;62:617-27.

\section{ACKNOWLEDGEMENTS}

Funding for this project was provided by the Stanford University Translational Research and Applied Medicine Program Pilot Grant (2018-2020), the Stanford INSPIRE Early Psychosis Clinic, the Stanford Schizophrenia Genetics Research Fund and $\mathrm{NIH}$ grant 5P50HG007735-05 (AEU). The Urban laboratory received funding from Mr. Bruce Blackie and from Dr. William Mclvor. DFL received support from the Walter E. Nichols MD Professorship in the School of Medicine, from Dr. William Mclvor, and from the Stanford Schizophrenia Genetics Research Fund. AK was supported by fellowship funding from T32 Fellowship funding from the National Institutes of Mental Health 5T32MH019938 and the MIRECC Fellowship. We would like to thank all the participants for their generous participation in this study.

\section{AUTHOR CONTRIBUTIONS}

$A K, J L, J B, D N, R O, D L$, and $A E U$ conceptualized the project, designed the study and methods. AK, JB, LA, and OL performed clinical assessments of participants. AK and $\mathrm{RP}$ performed the ddPCR assay. AK grew the BEC, performed immunofluorescence and permeability assay. CC and $\mathrm{JH}$ performed the cerebrospinal fluid collection. AK $E M, D L, J H$, and AEU wrote the manuscript and all the authors reviewed the manuscript and provided critical input $\mathrm{DL}, \mathrm{CS}$, and $\mathrm{AK}$ performed the statistical analysis. 


\section{COMPETING INTERESTS}

Dr. Jacob Hall receives speaker fees from Biogen. The remaining authors declare no competing interests.

\section{ADDITIONAL INFORMATION}

Supplementary information The online version contains supplementary material available at https://doi.org/10.1038/s41398-021-01583-5.

Correspondence and requests for materials should be addressed to Agnieszka Kalinowski or Alexander E. Urban.

Reprints and permission information is available at http://www.nature.com/reprints

Publisher's note Springer Nature remains neutral with regard to jurisdictional claims in published maps and institutional affiliations.
(C) Open Access This article is licensed under a Creative Commons Attribution 4.0 International License, which permits use, sharing, adaptation, distribution and reproduction in any medium or format, as long as you give appropriate credit to the original author(s) and the source, provide a link to the Creative Commons license, and indicate if changes were made. The images or other third party material in this article are included in the article's Creative Commons license, unless indicated otherwise in a credit line to the material. If material is not included in the article's Creative Commons license and your intended use is not permitted by statutory regulation or exceeds the permitted use, you will need to obtain permission directly from the copyright holder. To view a copy of this license, visit http://creativecommons. org/licenses/by/4.0/.

This is a U.S. government work and not under copyright protection in the U.S.; foreign copyright protection may apply 2021 